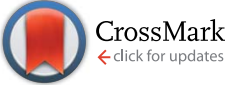

Cite this: Soft Matter, 2014, 10, 7337

\title{
Controlling the number of dendrimers in dendrimicelle nanoconjugates from 1 to more than $100 \dagger$
}

\author{
Junyou Wang, ${ }^{a}$ Ilja K. Voets, ${ }^{b}$ Remco Fokkink, ${ }^{c}$ Jasper van der Gucht ${ }^{c}$ \\ and Aldrik H. Velders*a
}

Herein, we present a facile strategy to controllably build up dendrimicelles by self-assembly of anionic PAMAM dendrimers with cationic-neutral diblock copolymers. We present a systematic study incorporating a full decade (0-9) of dendrimer generations, tracing the gradual variation from aggregates (G0 and G1) to self-assembled micelles (G2-G8), and an unidendrimer micelle structure (G9) by different scattering techniques (light and X-ray). The formed micelles (G2-G9) are spherical in shape with a hydrodynamic radius of about $25 \mathrm{~nm}$. Interestingly, the micellar size, structure and number of incorporated block copolymers are independent of the dendrimer generation (for G2 to G9), while the aggregation number of the dendrimers decreases from 108 to 1, and the stability of the micelles increases upon an increase in the dendrimer generation. Moreover, the micelles with lower generation dendrimers transform from spherical into worm-like structures upon an increase in the positive charge fraction (excess polymers) or ionic strength, while micelles with higher generation dendrimers do not show such a transition. This differential behavior is in-line with a change from a flexible configuration into rigid globular nanoparticles with increasing dendrimer generation. The reported systematic investigation of dendrimicelles comprising a full decade of dendrimer generations provides the basis for versatile strategies focused on building up new (multi)functional materials, e.g. by packing multiple types of dendrimers with different functional groups or encapsulated cargos controllably within one micelle.

Received 26th May 2014 Accepted 7th July 2014 DOI: $10.1039 / \mathrm{c} 4 \mathrm{sm} 01143 \mathrm{k}$ www.rsc.org/softmatter

\section{Introduction}

Dendrimers are three dimensional branched macromolecules with a well-defined architecture and size, highly adaptive surfaces and the ability to covalently or non-covalently bind a multitude of molecules. Dendrimers hold great promise in the design of smart materials, e.g. as carriers of therapeutic or diagnostic agents in medical applications. ${ }^{1,2}$ However, the potential applicability of single dendrimers is strongly limited by their constrained size and surface properties..$^{3-5}$ Therefore, supramolecular structures of dendrimers, in which size, shape, structure and functions are tunable, are widely investigated. ${ }^{6-10}$ In particular, core-shell dendrimicelles are believed to be an ideal platform in nanomedicine. ${ }^{11-13}$ The advantage of such dendrimicelles is that the cavity of dendrimers can be used for

${ }^{a}$ Laboratory of BioNanoTechnology, Wageningen University, Dreijenplein 6, $6703 \mathrm{HB}$ Wageningen, The Netherlands. E-mail: aldrik.velders@wur.nl

${ }^{b}$ Institute for Complex Molecular Systems and Laboratory of Macromolecular and Organic Chemistry, Eindhoven University of Technology, P.O. Box 513, 5600 MB Eindhoven, The Netherlands

'Laboratory of Physical Chemistry and Colloid Science, Wageningen University, Dreijenplein 6, 6703 HB Wageningen, The Netherlands

$\dagger$ Electronic supplementary information (ESI) available. See DOI: $10.1039 / \mathrm{c} 4 \mathrm{sm} 01143 \mathrm{k}$ encapsulation of hydrophilic functional cargos especially for controlled delivery and release applications in nanomedicine. This cannot be achieved with traditional micelles formed upon the assembly of amphiphilic molecules, as this results in a hydrophobic micellar core. The major challenge in this field is finding the design that allows for control over the structure, size, aggregation number and colloidal stability considering the branched structure and nano-scaled size of dendrimers. ${ }^{14,15}$

Polyamidoamines (PAMAMs) are among the most widely investigated dendrimers in self-assembly and medical applications, since they are commercially available and water-soluble and have demonstrated their efficacy in a variety of applications, including non-covalent molecular encapsulation. ${ }^{16,17}$ The well-defined number of surface charges of PAMAM dendrimers, easily obtained by specific terminating groups, provides an excellent driving force for self-assembly into supramolecular structures. ${ }^{18-22}$ In fact, PAMAM-based "unimolecular micelles" consisting of a single dendrimer modified with oppositely charged surfactants were reported about 15 years ago. ${ }^{23,24}$ More recently, Grohn et al. reported assembly of amine-terminated PAMAM dendrimers with $\mathrm{PMAA}_{170}-b-\mathrm{PEO}_{203}$ diblock copolymers, resulting in poorly controlled aggregates probably due to the insufficient protection by the PEO block. ${ }^{25}$ Herein, we introduce an improved design based on the well-established 
concept of complex coacervate core micelles (C3Ms), ${ }^{26}$ extended now to incorporate dendrimers within the coacervate core. We present the first systematic investigation of dendrimicelles comprising a full decade of dendrimer generations (0-9), showing excellent control over the self-assembly by incorporating from 1 to more than 100 dendrimers in equally sized micelles, which provides a solid basis for versatile strategies to construct new (multi)functional nanoconjugate materials.

\section{Results and discussion}

The formation of micelles in our study is based on electrostatic interactions between anionic carboxyl-terminated PAMAM dendrimers (Scheme 1a) and cationic-neutral diblock copolymers $\mathrm{P}_{2} \mathrm{MVP}_{128}-b-\mathrm{PEO}_{477}$ (Scheme $1 \mathrm{~b}$ ). Upon mixing the dendrimers and diblock copolymers in aqueous solution, the oppositely charged components associate building up a micellar core, surrounded by a corona of neutral PEO chains, leading to the formation of dendrimicelles (Scheme 1c). Addition of the P2MVP homopolymer to a G3 dendrimer solution induces a dramatic increase in light scattering intensity evidencing macroscopic phase separation, while addition of PEO to a G3 dendrimer solution does not lead to any significant change in scattering intensity. These observations indicate that micelle formation is indeed driven by the charged blocks and controlled by the neutral block. The diblock copolymer needs to be selected carefully as the charged groups, polymer length and the length ratio between the charged and neutral blocks strongly influence the final micelles. ${ }^{27}$ As a general rule, the neutral block should be at least 3 times as long as the charged block to restrict the phase separation to the colloidal domain to get stable micelles. The cationic block of our $\mathrm{P}_{2} \mathrm{MVP}_{128}-b$ $\mathrm{PEO}_{477}$ contains quaternized pyridine groups, such that the charge density is hence independent of pH. For PAMAM dendrimers, we chose $\mathrm{Na}_{2} \mathrm{CO}_{3} / \mathrm{NaHCO}_{3} \mathrm{pH} 10$ buffer, to deprotonate the carboxylic groups and the tertiary amines in the dendrimer core and branches. ${ }^{28,29}$ Under these conditions, the number of positive charges per polymer and the number of negative charges per dendrimer are identical to the numbers of pyridinium and carboxylic groups, respectively.

We study the mixture of PAMAM dendrimer and diblock copolymers by means of light scattering (LS); the occurrence of micelles should give an enhancement of scattered light. Upon addition of the $\mathrm{P}_{2} \mathrm{MVP}_{128}-b-\mathrm{PEO}_{477}$ copolymer to a solution containing G0 PAMAM (4 -COONa), soluble aggregates (dendrimer-decorated polymer chains, vide infra) are formed. Addition of a block copolymer to G1 PAMAM (8 - COONa) probably yields floppy irregular aggregates, as suggested by the low scattering intensity and relatively large hydrodynamic size, in contrast to the well-defined micelles that we find for dendrimers of generations 2 to 9 . In view of these results, we do not pursue the study of G0 and G1 any further, but concentrate on G2-G9. The formed micelles at generations 2 to 9 are called complex coacervate core micelles (C3Ms), ${ }^{26}$ and are represented as Gn-C3Ms with $\mathrm{Gn}$ indicating the dendrimer generation. ${ }^{30} \mathrm{G0}$ and G1 PAMAM dendrimers do not form micelles, which is consistent with a previous study on coacervate micelles from linear polyelectrolytes, demonstrating that micelles form only

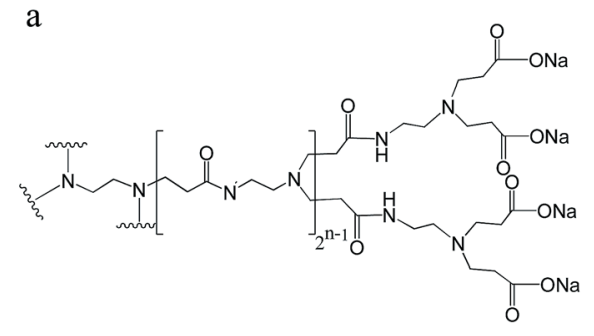

b
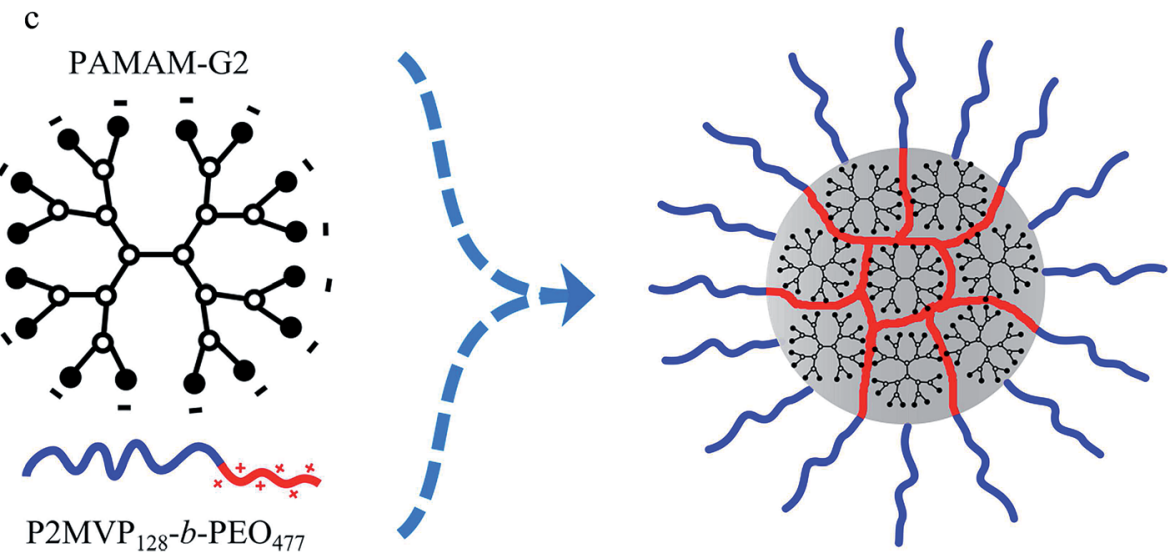

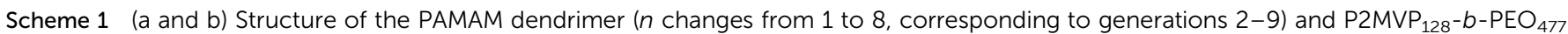
diblock copolymer. The open circle indicates the interior $\mathrm{N}$ atom and the filled circle represents the surface group -COONa. (c) Formation of micelles from PAMAM-G2 and P2MVP $128-b-\mathrm{PEO}_{477}$ diblock copolymer. 
when the polymer chain length exceeds a certain critical length. ${ }^{27,31}$

Below we focus on different aspects of Gn-C3Ms with PAMAM dendrimers of $2 \leq n \leq 9$. First, LS titrations are discussed and used to determine the optimal charge fraction $f+$ for micellization of the Gn-C3Ms. Here $f^{+}$is defined as $f+=\frac{n^{+}}{n^{+}+n^{-}}$where $n^{+}$and $n^{-}$are the numbers of positive and negative charges. Additional analyses of the LS data and SAXS (small angle X-ray scattering) measurements provide the size, shape, molar mass, aggregation number and stability of the formed micelles. Further analysis of the titration curves shows interesting trends as a function of dendrimer generation, both at lower charge ratios (excess of dendrimer) and higher charge ratios (excess of polymer), as well as at different salt concentrations. We summarize the results in a general diagram which schematically depicts the different structures and boundaries observed as a function of dendrimer generation and (mixing) charge fraction.

\section{Preferred micellar composition}

Fig. 1 shows LS titration curves corresponding to stepwise addition of $\mathrm{P}_{2} \mathrm{MVP}_{128}-b-\mathrm{PEO}_{477}$ diblock copolymers to aqueous dendrimer solutions, for PAMAM dendrimers of generation 2 to 9. The light scattering intensity initially increases and reaches a maximum corresponding to the preferred micellar composition (PMC) where charge stoichiometry is satisfied. ${ }^{27}$ The charge fraction at PMC is usually close to 0.5 for micelles of linear polyelectrolytes. ${ }^{26}$ For the dendrimicelles reported here, we find again the PMC at $f+\sim 0.5$ for G2 and G3, while it shifts to $f+<0.5$ with increasing dendrimer generation (see Table 1), indicating that less positive charge (viz. diblock copolymer) is required to reach the PMC. As the PMC $f+$ is calculated based on 'perfect' dendrimers with theoretical molecular weight and number of end groups, the deviations of PMC $f+$ from 0.5 may be attributed to the presence of defects and other impurities, e.g. dimers and trimers, in the specific dendrimer batch. The number of defects and amount of impurities are known to increase with increasing dendrimer generation; ${ }^{32,33}$ overall, this should result in a net reduction of the charge per weight, which means that there are
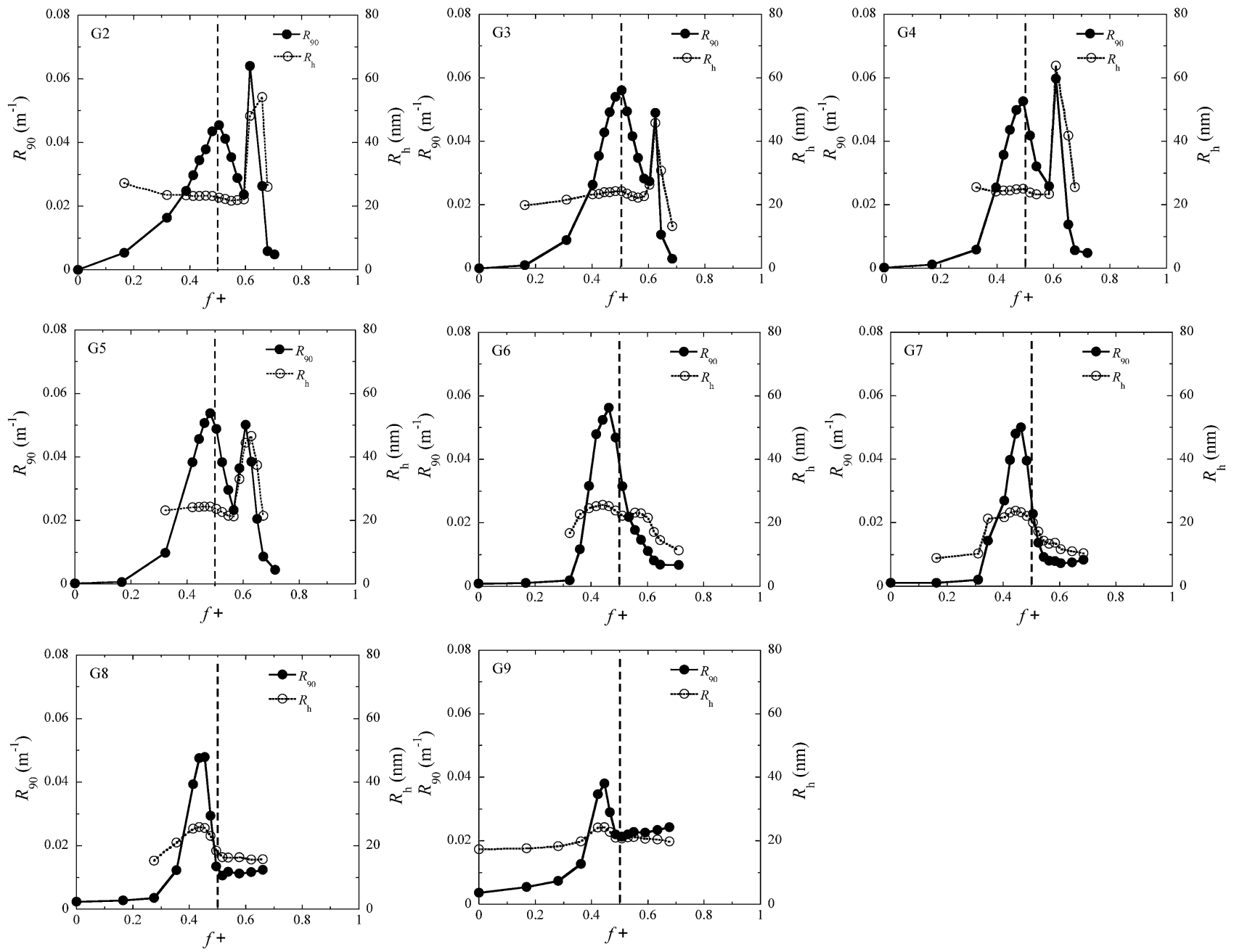

Fig. 1 Light scattering titration of PAMAM dendrimers at different generations with $\mathrm{P}_{2} \mathrm{MVP}_{128}-b-\mathrm{PEO}_{477}$. The light scattering intensity $R_{90}$ and hydrodynamic radius $R_{\mathrm{h}}$ are plotted as a function of positive charge fraction $f+$. The dashed line shows the position of the theoretical $f+=0.5$, and deviations from that line reflect the presence of defects and impurities in the dendrimer batch. 
Table 1 Summary of parameters of PAMAM dendrimers and the formed micelles upon co-assembly with $\mathrm{P}_{2} \mathrm{MVP}_{128}-b-\mathrm{PEO}_{477}$

\begin{tabular}{|c|c|c|c|c|c|c|c|c|c|}
\hline Generation & $(N-)^{a}$ & $\operatorname{PMC} f+^{b}$ & $M_{\text {micelle }}^{c} \times 10^{6} \mathrm{~g} \mathrm{~mol}^{-1}$ & $R_{\mathrm{h}}(\mathrm{nm})$ & $N_{\text {PAMAM }^{d}}$ & $N_{\text {Polymer }}$ & $R_{\text {core }}(\mathrm{nm})$ & $H_{\text {shell }}(\mathrm{nm})$ & $C_{\mathrm{s}, \mathrm{cr}} \pm 10(\mathrm{mM})$ \\
\hline 2 & 16 & 0.50 & 1.10 & 23.9 & 108 & 16 & 10.9 & 12.8 & 155 \\
\hline 3 & 32 & 0.50 & 1.16 & 24.3 & 56 & 16 & 13.3 & 12.1 & 206 \\
\hline 5 & 128 & 0.48 & 1.19 & 25.5 & 15 & 16 & 13.5 & 12.5 & 309 \\
\hline 6 & 256 & 0.46 & 1.20 & 25.1 & 8 & 16 & 12.4 & 12.1 & 347 \\
\hline 7 & 512 & 0.46 & 1.23 & 23.9 & 4 & 16 & 11.2 & 11.5 & 390 \\
\hline
\end{tabular}

${ }^{a} N$ represents theoretical surface numbers. ${ }^{b}$ PMC charge fraction $f+$ was calculated based on the theoretical numbers of dendrimers to indicate the $f+$ shifts. ${ }^{c}$ Micellar properties, micellar mass $M_{\text {micelle, }}$, hydrodynamic radius $R_{\mathrm{h}}$, the micellar core radius $\left(R_{\text {core }}\right)$, shell thickness $\left(H_{\text {shell }}\right)$ and critical salt concentration $C_{\mathrm{s}, \mathrm{cr}}$ are obtained by light and X-ray scattering measurements. ${ }^{d}$ Aggregation numbers, $N$, are integer values from calculation as given in the ESI.

less negative charges per sample than expected from theoretical calculations. Since the determined PMC indicates the actual (charge) stoichiometry, the observed deviation from 0.5 has been taken into account in the calculations of the aggregation number (see ESI $\dagger$ for details on computation).

\section{Size, shape, aggregation number, and salt stability of Gn- C3Ms}

Fig. 2a shows the micellar mass and hydrodynamic radius of Gn-C3Ms (G2-G9) at the PMC, obtained from the light scattering experiments, as a function of dendrimer generation (see also ESI, Fig. S1†). Remarkably, Gn-C3Ms formed from different dendrimer generations barely differ in micellar mass and hydrodynamic radius, which are approximately $1.2 \times 10^{6} \mathrm{~g}$ $\mathrm{mol}^{-1}$ and $25 \mathrm{~nm}$, respectively. CONTIN analyses of the DLS data indicate that there is one dominant distribution of particle sizes of Gn-C3Ms irrespective of dendrimer generation (ESI, Fig. S3†). Small-angle X-ray scattering (Fig. 2b) confirms that Gn-C3Ms of $2 \leq n \leq 9$ are core-shell structures with spherical shape. We selected a polydisperse core-shell model to describe
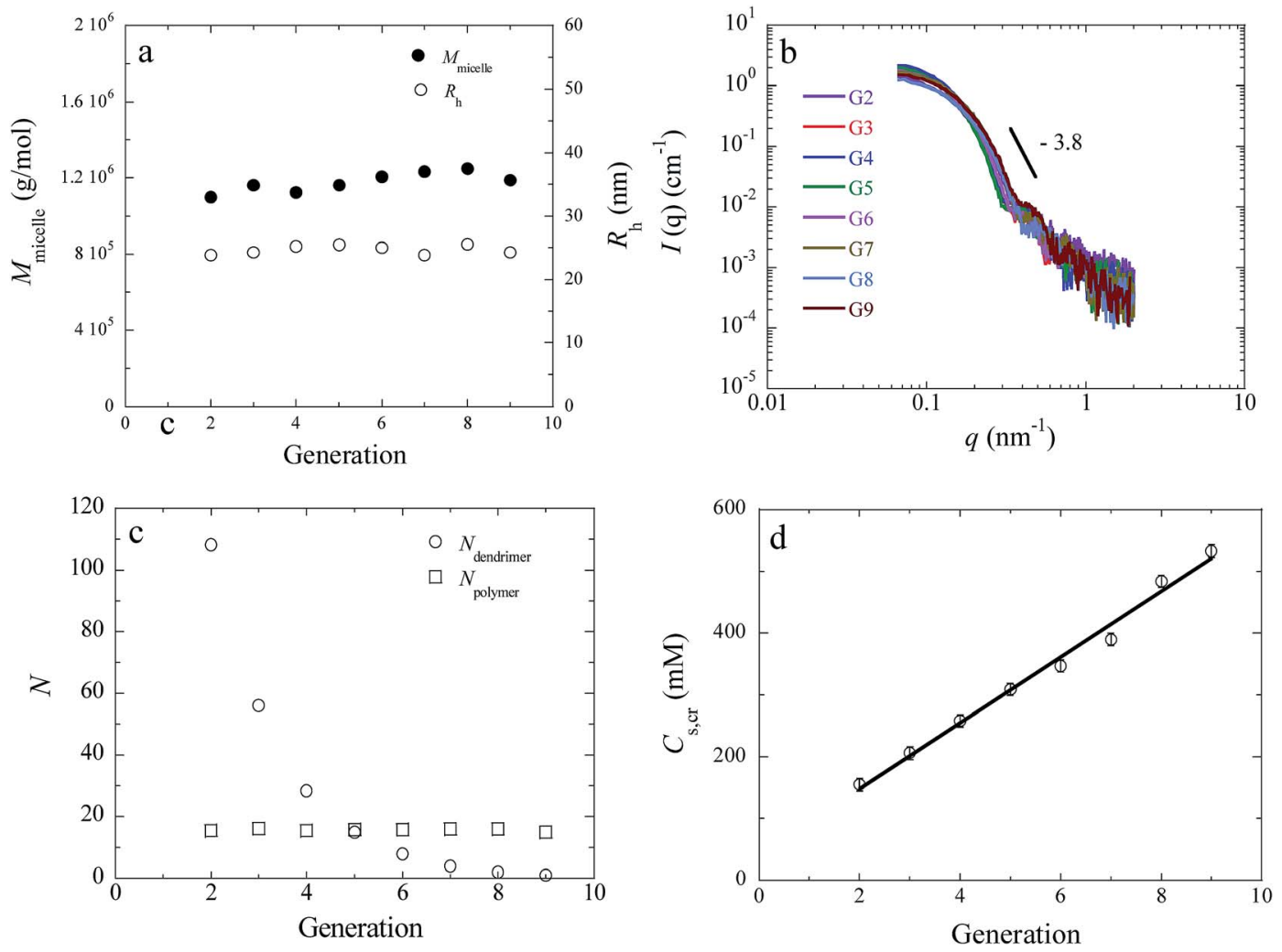

Fig. 2 Micellar mass $M_{\text {micelle }}$ and hydrodynamic radius $R_{\mathrm{h}}$ (a), SAXS profiles (b), aggregation number $N$ (c), and critical salt concentration $C_{\mathrm{s}, \mathrm{cr}}$ (d) of $\mathrm{Gn}-\mathrm{C} 3 \mathrm{Ms}$ incorporating PAMAM dendrimers of different generations in the range G2-G9. Micelles are prepared in $\mathrm{NaHCO}_{3} / \mathrm{Na}_{2} \mathrm{CO}_{3}$ buffer (20 $\mathrm{mM}, \mathrm{pH} 10)$ at the PMC. 
the experimental data (ESI, Fig. S4 $\dagger$ ) and found a core radius, $R_{\text {core }}=12 \pm 1 \mathrm{~nm}$ and shell thickness, $H_{\text {corona }}=12 \pm 1 \mathrm{~nm}$ for Gn-C3Ms of all generations from 2 to 9 (Table 1). Clearly, the dendrimer size and surface charge have no effect on the size and structure of Gn-C3Ms in the studied generation range of G2 to G9. This is analogous to a previous study on C3Ms of poly(acrylic acid) (PAA) and $\mathrm{P}_{2} \mathrm{MVP}_{128}-b-\mathrm{PEO}_{477}$ diblock copolymers, where constant-size C3Ms were formed as long as $N_{\text {PAA }}$ does not exceed a certain critical chain length, $\sim 2000 \mathrm{AA}$ units. ${ }^{34}$ G9 PAMAM dendrimers contain 2048 -COONa surface groups without considering defects. Hence, Gn-C3Ms of $n \leq 9$ show constant size, resembling the behaviour of C3Ms based on linear PAA polymers.

Since the dendrimer size and surface charge do increase with increasing dendrimer generation, the observed uniformity in micellar size from all Gn-C3Ms implies that the aggregation number of the dendrimers must decrease with increasing generation. This is equivalent to the condition, $n^{+}=n^{-}$, or $N_{\mathrm{p}} Z_{\mathrm{p}}$ $=N_{\mathrm{d}} Z_{\mathrm{d}}$, where $N_{\mathrm{p}}$ and $N_{\mathrm{d}}$ are the numbers of cationic blocks and dendrimers per micelle and $Z_{\mathrm{p}}$ and $Z_{\mathrm{d}}$ are the charge numbers per molecule. We determined the aggregation numbers of dendrimers and polymers from partial Zimm analyses of the static light scattering data (ESI, Fig. S1 $\dagger$ ) which are presented in Fig. 2c (see also Table 1). Each micelle contains on average 16 block copolymers for Gn-C3Ms of all generations. The dendrimer aggregation number, on the other hand, decreases roughly by a factor of 2 with each increment in the generation number, which is in line with a two-fold increase in dendrimer surface charge upon an increase in the generation number. Eventually, G2-C3Ms contain 108 dendrimers whilst G9-C3Ms contain only 1 dendrimer per micellar core and as such can be regarded as unidendrimer micelles. ${ }^{35}$

These findings may be interpreted as follows. For all stable micelles, the size is controlled by a balance between the interfacial tension of the (complex) core and the osmotic pressure in the corona, which essentially depends on the length of the neutral (PEO) blocks. ${ }^{36,37}$ Since these two parameters do not depend on dendrimer generation, the micellar size and the number of diblocks per micelle, is constant and independent of dendrimer generation. Here, $\mathrm{P}_{2} \mathrm{MVP}_{128}-b-\mathrm{PEO}_{477}$ diblock
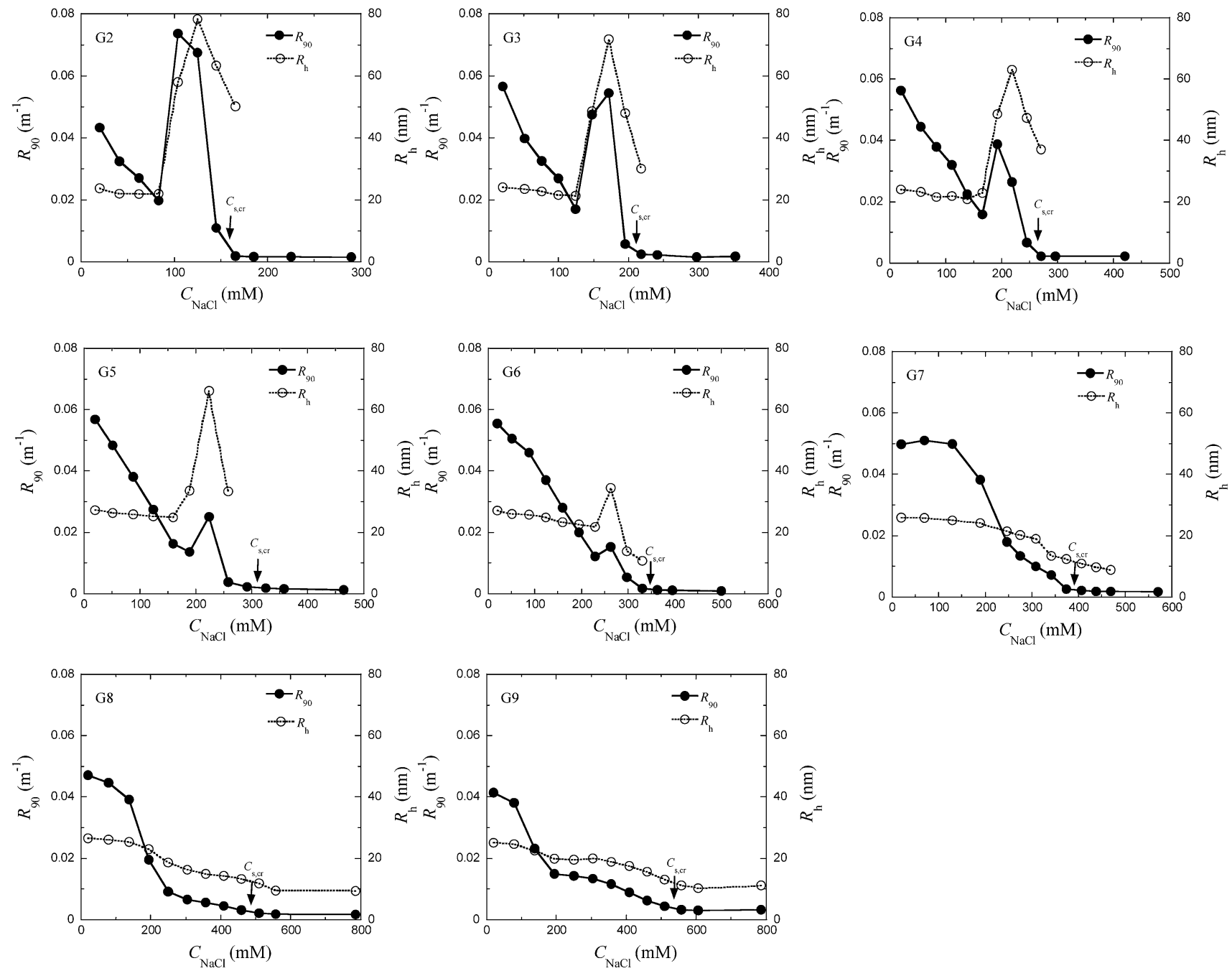

Fig. 3 Variation of light scattering intensity $R_{90}$ and hydrodynamic radius $R_{\mathrm{h}}$ of Gn-C3Ms with increasing salt concentration, which is the sum of $\mathrm{NaCl}$ concentration added and the concentration of buffer viewed as 1-1 type electrolyte. 
copolymers build up a cage of constant size fixed by the number of block copolymers, hence, cationic groups, per micelle. To fill the cage and fully compensate the block copolymer charge, either many small dendrimers with a low surface charge are required, or alternatively, few large dendrimers with a high surface charge. In other words, the dendrimer generation regulates the number of dendrimers incorporated into GnC3Ms of constant size.

The critical salt concentration, $C_{\mathrm{s}, \mathrm{cr}}$, reflects the salt stability of micelles formed upon co-assembly of oppositely charged macromolecules. ${ }^{36,37}$ We obtain the $C_{\mathrm{s}, \mathrm{cr}}$ of Gn-C3Ms from salt titration curves (Fig. 3) by taking the average of the two boundary points where the intensity drops to the baseline value. About $20 \mathrm{mM}$ salt is added per titration step, so the error is estimated to be $\pm 10 \mathrm{mM}$. Fig. $2 \mathrm{~d}$ shows a linear increase in $C_{\mathrm{s}, \mathrm{cr}}$ with increasing dendrimer generation. In summary, Gn-C3Ms $(2 \leq n \leq 9)$ are spherical micelles with a core-shell structure and constant size, whilst the number of dendrimers inside the micellar core as well as salt stability are tuneable with the dendrimer generation as the control parameter.

\section{Morphology of Gn-C3Ms}

As C3Ms typically respond to changes in ionic strength, ${ }^{38,39}$ we investigated the light scattering intensity and hydrodynamic radius of PAMAM dendrimicelles upon increasing salt concentration. Fig. 3 shows that for all Gn-C3Ms both the intensity and $R_{\mathrm{h}}$ decrease upon increasing salt concentration, which is due to an increase in the critical micellization concentration, CMC, and a decrease of the micellar aggregation number with increasing ionic strength..$^{39}$ Interestingly, Gn-C3Ms of low $(\leq \mathrm{G} 6)$ and high generation ( $>\mathrm{G} 6)$ respond differently to an increase in ionic strength. The light scattering intensity decreases gradually upon an increase in ionic strength for Gn-C3Ms of $n>6$, while a peak in scattering intensity emerges at intermediate ionic strength in solutions of Gn-C3Ms with $n \leq 6$. The hydrodynamic radius of the Gn-C3Ms follows the same trend: $R_{\mathrm{h}}$ decreases in a monotonous fashion upon increasing ionic strength for $n>6$, while assemblies with a larger $R_{\mathrm{h}}$ appear at intermediate ionic strength for $n \leq 6$. Similarly, in the composition titrations in Fig. 1 we find that both the scattering intensity and $R_{\mathrm{h}}$ decrease gradually with increasing $f+$ for $n \geq 6$, while a peak in both intensity and hydrodynamic radius emerges for $n \leq 5$. Several studies have shown that low generation $(n \leq 4)$ PAMAM dendrimers are soft structures with compressible configurations, while G7 and higher generation dendrimers behave like uncompressible hard spheres; G5 and G6 constitute the boundary from soft to hard. ${ }^{40,41}$ Since our titration studies exhibit the exact same tipping point $(n \sim 5-6)$, we attribute the different decay behaviors in the composition and salt titrations for low $(\leq 5)$ and high $(\geq 6)$ generation Gn-C3Ms to the configurational transition of dendrimers from soft to hard, respectively.

We decided to further investigate the origin of the side maximum in the composition titrations (Fig. 1) and the peak observed in the salt titrations (Fig. 3). A morphological transition from spherical to wormlike micelles has been identified in a previous study on linear polyelectrolyte coacervate micelles as the reason for the peak in light scattering intensity and $R_{\mathrm{h}}$ at high $f+$ in composition titrations and high ionic strength in salt titrations. ${ }^{34,42}$ To evaluate whether dendrimer micelles yield similar structures, angular-dependent dynamic light scattering experiments were carried out on G3-C3Ms at $f+0.5(20 \mathrm{mM}$, $\mathrm{Na}_{2} \mathrm{CO}_{3} / \mathrm{NaHCO}_{3}$ buffer, control sample) and $f^{+}=0.67$ (side maximum) and at $f+0.5$ in the same buffer with $160 \mathrm{mM} \mathrm{NaCl}$ added. CONTIN analyses reveal multimodal distributions with a non-negligible dependence of the average hydrodynamic radius for G3-C3Ms at $f+=0.67$ and 0.5 in the salt buffer (Fig. 4). By contrast, the control sample with G3-C3Ms at $f+=0.5$ reveals a monomodal distribution with a hydrodynamic radius independent of the scattering angle. These findings strongly suggest that G3-C3Ms are rather well-defined spherical micelles with undetectable rotational motion at the PMC and low ionic strength, which transform into elongated (e.g., worm-like) or large, polydisperse spherical objects upon addition of excess block copolymer or $\mathrm{NaCl}$. Depolarized light scattering measurements were performed to discriminate between the formation of worm-like micelles and polydisperse spherical aggregates. The incoming light is vertically polarized in DLS, and the scattered light by a spherical object is vertically polarized as well because of isotropic rotation. By contrast, anisotropic structures, such as worms or rods, scatter both vertically
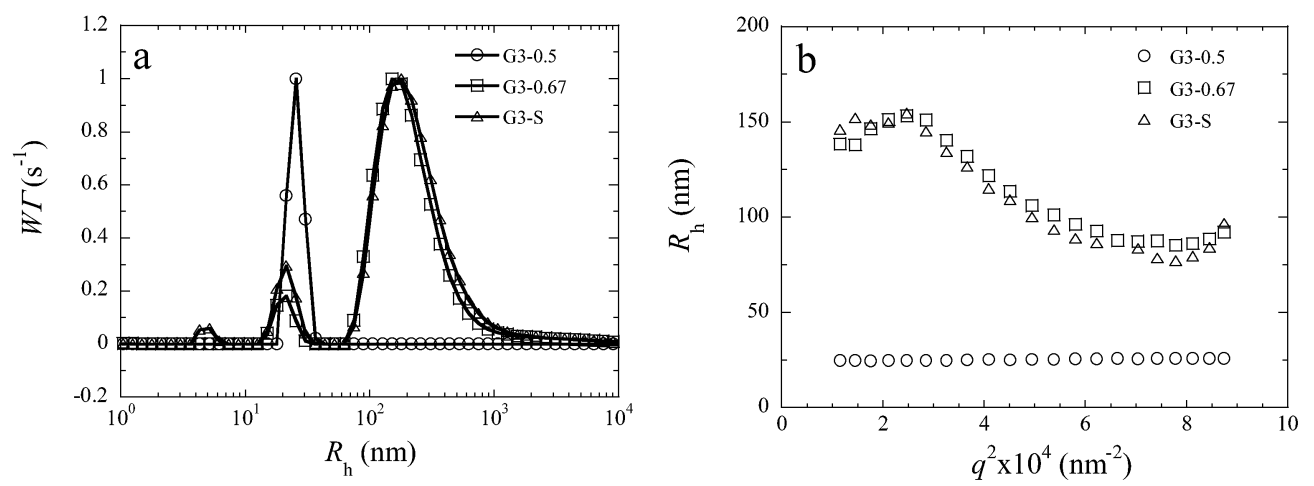

Fig. 4 Size distribution $\left(\mathrm{a}, \theta=90^{\circ}\right)$ and angular dependence of the average hydrodynamic radius $\left(\mathrm{b}, 40^{\circ} \leq \theta \leq 140^{\circ}\right)$ of $\mathrm{G} 3-\mathrm{C} 3 \mathrm{Ms}$ at $f+0.5,0.67$ and at $f+0.5$ with $160 \mathrm{mM} \mathrm{NaCl}$ in buffer solution. 
$\left(I_{\mathrm{vv}}\right)$ and horizontally $\left(I_{\mathrm{vh}}\right)$ polarized light. The depolarized ratio $\Delta_{\mathrm{vh}}$ (ESI, $\dagger$ Experimental section, eqn (8)) reflects the degree of anisotropy scaled with both the number and length of the anisotropic structures in solution. ${ }^{43,44}$ The $\Delta_{\mathrm{vh}}$ of G3-C3Ms in the control sample $f+0.5$ was $7.1 \times 10^{-4}$, whilst $f+0.67$ and $f+$ 0.5 at $160 \mathrm{mM} \mathrm{NaCl}$ were found to be $5.7 \times 10^{-3}$ and $3.6 \times 10^{-3}$. These ratios are close to the $\Delta_{\mathrm{vh}}$ of spherical and wormlike micelles of linear polyelectrolyte micelles, ${ }^{34,42}$ confirming that the G3-C3Ms at $f+0.5$ are spherical, whereas the larger aggregates at the side maximum in composition and salt titrations indeed are asymmetric structures formed only for the lower generation PAMAM dendrimicelles. Apparently, micelles change from a spherical to wormlike morphology upon increasing $f^{+}$and salt concentration during the composition and salt titrations. The transition seems to require sufficiently soft dendrimers (low generation) to allow for the necessary deformation of the micellar core and rearrangement into wormlike structures. Micelles with high generation dendrimers with more rigid configuration are apparently too stiff to be deformed and rearranged, so no transition from spherical to wormlike aggregates occurs. In addition, the morphological transition may require a critical number of dendrimers per micelle, as it is more difficult to rearrange a few high generation dendrimers into a wormlike micelle than many of low generation dendrimers. Alternatively, the exchange dynamics of the polymer chains comprising the micelles may also impact the morphological transition. Higher generation dendrimers bind more strongly to $\mathrm{P}_{2} \mathrm{MVP}_{128}-b-\mathrm{PEO}_{477}$ diblock copolymers, which may hamper relaxation towards equilibrium structures and impede structural deformation.

\section{Non-stoichiometric compositions}

In the above, we have discussed the structures formed at the PMC and second maximum in the composition titration curve (Fig. 1). Now we will focus on compositions away from the PMC; that is, $f^{+}<0.5$ and $f^{+}>0.5$ except for the range of second maximum. For G2, the light scattering intensity increases upon adding $\mathrm{P}_{2} \mathrm{MVP}_{128}-b-\mathrm{PEO}_{477}$ diblock copolymers to PAMAM solution. The hydrodynamic radius does not vary while the intensity increases with increasing polymer concentration, which means that the micelles are formed immediately after adding the polymer; increasing the polymer concentration further only increases the number of micelles but does not alter the micellar size and structure. This immediate increase in intensity becomes less strong with increasing generation, and for the generations 6 and higher, no increase in intensity is observed before $f+\sim 0.3$. Probably, $\mathrm{P}^{2} \mathrm{MVP}_{128}-b-\mathrm{PEO}_{477}$ polymers can easily grab smaller and more flexible dendrimers into micelles for the lower PAMAM generations; for higher generation dendrimers with stiff configuration and more surface charges, polymers likely first attach on a single dendrimer when starting the titration and in consecutive titration steps cross link multiple dendrimers into micelles with further increasing block copolymer amounts.

In the range where $f+$ is higher than the PMC, the intensity goes down with increasing $f+$. Gn-C3Ms from low generation
( $\leq$ G5) show constant size/hydrodynamic radius, indicating that only the number of micelles decreases with increasing polymer concentration. We have found that the intensity and radius of micelles show a dramatic increase just around $f+0.65$, which is due to the transition from sphere to elongated structures, as proven above by angular dependent and depolarized DLS measurements. The intensity drops sharply after this bump to very low values, suggesting only small molecules to be present in solution. Differently, for micelles derived from high generation dendrimers $(\geq \mathrm{G} 6)$, the hydrodynamic radius drops with increasing $f_{+}$after PMC, which means that the aggregation number of micelles decreases with increasing polymer concentration. Interestingly, the intensity reaches a plateau, and the value of the intensity of the plateau increases with increasing generation from 6 to 9 , consistent with the increase in the size of the final structures from G6 to G9. The size in the plateau range is between the sizes of the free dendrimer and the real micelles, indicating unidendrimer structures to be present in solution with single dendrimers covered by polymers.

In a composition titration of a unidendrimer micelle, G9C3M, polymers aggregate on the surface of the single dendrimer until it is saturated at PMC, after which the micelles remain intact because excess polymer cannot dissociate the formed micelles into smaller complexes. We therefore expect a gradual increase in the intensity before the PMC is reached and a constant intensity and radius after the PMC. Experimentally, however, we do find a peak in the intensity around the PMC (Fig. 1). This may be because a commercial sample of G9 PAMAM dendrimer contains a significant percentage of lower generation dendrimeric structures, as reported previously for G5 PAMAM dendrimers. ${ }^{33}$ These smaller $(<\mathrm{G} 9)$ dendrimers in a G9-C3M dendrimicelle sample will self-assemble with polymers and undergo a formation-dissociation process with increasing polymer concentration, leading to an increase and decay of the intensity as is clear from the DLS curves of the lower generations. ${ }^{45}$

\section{State diagram of dendrimicelles}

In summary, upon mixing anionic PAMAM dendrimers with cationic-neutral diblock copolymers $\mathrm{P}_{2} \mathrm{MVP}_{128}-b-\mathrm{PEO}_{477}$ in aqueous solution, various aggregates form depending on the charge fraction and dendrimer generation as schematically depicted in the state diagram in Fig. 5. G0 and G1 dendrimers cannot form micellar structures at all. For clarification, we normalized all the PMC charge fractions of G2 to G9 to the value of $f+=0.5$; the corresponding boundaries as observed in the LS titration curves shown in Fig. 1 have been adapted accordingly. The area bracketed by the blue lines corresponds to the regime wherein C3Ms dominate; that is, the boundary corresponds to the composition at half the intensity of the PMC. At $f+$ values smaller than the PMC, low generation dendrimers coexist with the formed micelles, while high generation dendrimers coexist with dendrimers decorated partly by the polymer. The number of micelles increases with increasing $f+$ and reaches a maximum around the PMC. The size and mass of Gn-C3Ms at the PMC are hardly dependent on dendrimer generation, while the 


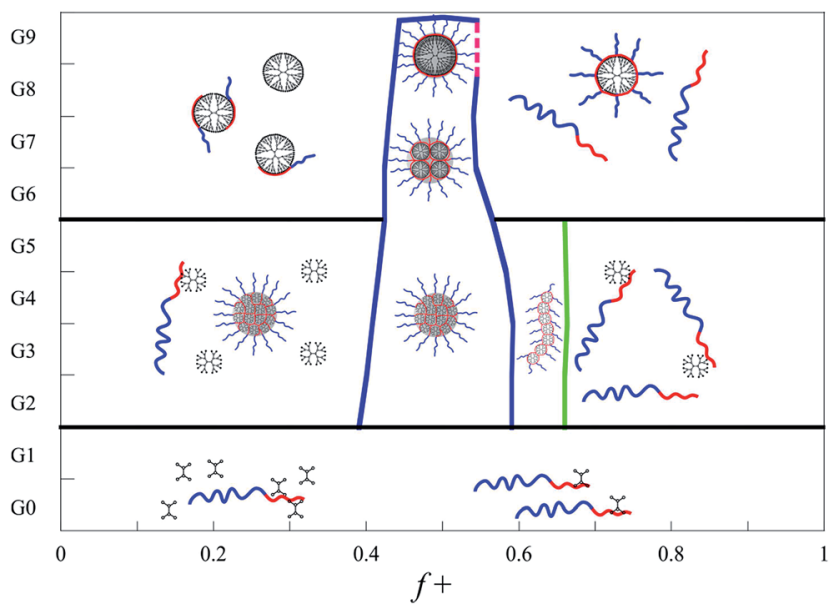

Fig. 5 General state diagram of the dominant structures formed by PAMAM dendrimer and $\mathrm{P}_{2} \mathrm{MVP}_{128}-b-\mathrm{PEO}_{477}$ polymers upon varying mixing charge fraction and dendrimer generation. For micelles around PMC, the dendrimer numbers are not scaled to real aggregation number and only highlight the decay trends with increasing dendrimer generation.

dendrimer (aggregation) number strongly decreases with increasing dendrimer generation. The area delineated by the green line indicates the presence of elongated structures instead of spherical micelles observed for Gn-C3Ms of low generation dendrimers. These large structures dissociate upon further increasing $f+$ into small complexes of polymer chains decorated by one or several dendrimers. Wormlike micelles do not form in solutions with high generation dendrimers at any composition. Under extreme non-stoichiometric conditions $(f+$ $\gg$ PMC), single dendrimers covered fully by polymers coexist with free polymers. There should be no decay on aggregation numbers for unidendrimer G9-C3Ms after PMC (indicated as the red dashed line in Fig. 5), so the formed micelles do not change and only the free polymer increases with increasing $f+$.

\section{Conclusion}

We have demonstrated a facile strategy to control the packing of PAMAM dendrimers, from generations 2 to 9, into well-defined micellar structures, coined dendrimicelles. The micellization is driven by the electrostatic interaction between carboxyl-terminated PAMAM dendrimers and cationic-neutral diblock copolymers $\mathrm{P}_{2} \mathrm{MVP}_{128}-b-\mathrm{PEO}_{477}$. The formed micelles are spherical in shape with a hydrodynamic radius of about $25 \mathrm{~nm}$, and the micellar size, structure and number of incorporated block copolymers are independent of the dendrimer generation, while the aggregation number of the dendrimers decreases and the salt stability of the micelles increases upon an increase in the dendrimer generation. Remarkably, the micelles from lower generation dendrimers transfer from spherical to worm-like structures upon increasing positive charge fraction after the PMC or above a certain ionic strength value, while micelles with higher generation dendrimers do not show such a transition. This different behavior is in-line with a change of flexible configuration to rigid globular nanoparticles with increasing dendrimer generation. The presented Gn-C3M system promises to be a general strategy for other dendrimer and block copolymer systems allowing for systematic studies revealing periodic trends in the physical-chemical properties of the polymer building block nanoscale assemblies. ${ }^{4-48}$ Moreover, the high uniformity in structure and size, with tunable dendrimer aggregation number and salt stability provide great potential for developing multifunctional materials, considering the widespread applications of single dendrimers with different functional groups, e.g. fluorophores, ${ }^{49}$ or encapsulated cargos, e.g. nanoparticles, ${ }^{50-52}$ that can now be packed controllably in one micelle structure, particularly for nanomedical applications where controlled aggregation size and stoichiometry is required.

\section{References}

1 S. H. Medina and M. E. H. El-Sayed, Chem. Rev., 2009, 109, 3141-3157.

2 M. A. Mintzer and M. W. Grinstaff, Chem. Soc. Rev., 2011, 40, 173-190.

3 A. Sousa-Herves, E. Fernandez-Megia and R. Riguera, Chem. Commun., 2008, 3136-3138.

4 J. Khandare, M. Calderon, N. M. Dagia and R. Haag, Chem. Soc. Rev., 2012, 41, 2824-2848.

5 W. C. She, K. Luo, C. Y. Zhang, G. Wang, Y. Y. Geng, L. Li, B. He and Z. W. Gu, Biomaterials, 2013, 34, 1613-1623.

6 V. V. Narayanan and G. R. Newkome, Top. Curr. Chem., 1998, 197, 19-77.

7 S. C. Zimmerman and L. J. Lawless, Top. Curr. Chem., 2001, 217, 95-120.

8 J. M. J. Frechet, Proc. Natl. Acad. Sci. U. S. A., 2002, 99, 47824787.

9 P. J. Gittins and L. J. Twyman, Supramol. Chem., 2003, 15, $5-23$.

10 D. K. Smith, A. R. Hirst, C. S. Love, J. G. Hardy, S. V. Brignell and B. Q. Huang, Prog. Polym. Sci., 2005, 30, 220-293.

11 M. J. Liu, K. Kono and J. M. J. Frechet, J. Controlled Release, 2000, 65, 121-131.

12 R. Haag, Angew. Chem., Int. Ed., 2004, 43, 278-282.

13 S. Svenson and D. A. Tomalia, Adv. Drug Delivery Rev., 2005, 57, 2106-2129.

14 D. E. Discher and R. D. Kamien, Nature, 2004, 430, 519-520. 15 X. H. Xu, H. Yuan, J. Chang, B. He and Z. W. Gu, Angew. Chem., Int. Ed., 2012, 51, 3130-3133.

16 D. Astruc, E. Boisselier and C. Ornelas, Chem. Rev., 2010, 110, 1857-1959.

17 C. C. Lee, J. A. MacKay, J. M. J. Frechet and F. C. Szoka, Nat. Biotechnol., 2005, 23, 1517-1526.

18 N. Tomioka, D. Takasu, T. Takahashi and T. Aida, Angew. Chem., Int. Ed., 1998, 37, 1531-1534.

19 B. L. Frankamp, A. K. Boal and V. M. Rotello, J. Am. Chem. Soc., 2002, 124, 15146-15147.

20 D. A. Tomalia, H. M. Brothers, L. T. Piehler, H. D. Durst and D. R. Swanson, Proc. Natl. Acad. Sci. U. S. A., 2002, 99, 50815087. 
21 F. Grohn, Soft Matter, 2010, 6, 4296-4302.

22 M. L. Ainalem and T. Nylander, Soft Matter, 2011, 7, 45774594.

23 V. Chechik, M. Q. Zhao and R. M. Crooks, J. Am. Chem. Soc., 1999, 121, 4910-4911.

24 G. Caminati, N. J. Turro and D. A. Tomalia, J. Am. Chem. Soc., 1990, 112, 8515-8522.

25 F. Reinhold, U. Kolb, I. Lieberwirth and F. Grohn, Langmuir, 2009, 25, 1345-1351.

26 I. K. Voets, A. de Keizer and M. A. Cohen Stuart, Adv. Colloid Interface Sci., 2009, 147-48, 300-318.

27 S. van der Burgh, A. de Keizer and M. A. Cohen Stuart, Langmuir, 2004, 20, 1073-1084.

28 N. Miura, P. L. Dubin, C. N. Moorefield and G. R. Newkome, Langmuir, 1999, 15, 4245-4250.

29 D. Cakara, J. Kleimann and M. Borkovec, Macromolecules, 2003, 36, 4201-4207.

30 Dendrimer structures contain three main components: the core, the repeat units and surface groups. The number of repeat units indicates the dendrimer generation and determines the number of surface groups. The carboxylterminated PAMAM dendrimers have the same amount of repeat units and surface groups with amine-terminated PAMAM dendrimers under the same generation. Hence, instead of using a half-generation number, we use an even number to represent the generation of carboxyl-terminated PAMAM dendrimers.

31 Y. Yan, N. A. M. Besseling, A. de Keizer, A. T. M. Marcelis, M. Drechsler and M. A. Cohen Stuart, Angew. Chem., Int. Ed., 2007, 46, 1807-1809.

32 D. G. Mullen, A. Desai, M. A. van Dongen, M. Barash, J. R. Baker and M. M. B. Holl, Macromolecules, 2012, 45, 5316-5320.

33 M. A. van Dongen, A. Desai, B. G. Orr, J. R. Baker Jr and M. M. Banaszak Holl, Polymer, 2013, 54, 4126-4133.

34 H. M. van der Kooij, E. Spruijt, I. K. Voets, R. Fokkink, M. A. Cohen Stuart and J. van der Gucht, Langmuir, 2012, 28, 14180-14191.

35 G9-C3M contains one dendrimer and $16 \mathrm{P}_{2} \mathrm{MVP}_{128}-b-\mathrm{PEO}_{477}$ polymers, which is not a real unimolecular structure. So we use "unidendrimer micelles" instead of "unimolecular micelles".
36 J. Y. Wang, M. A. C. Stuart, A. T. M. Marcelis, M. ColombDelsuc, S. Otto and J. van der Gucht, Macromolecules, 2012, 45, 7179-7185.

37 J. Y. Wang, A. H. Velders, E. Gianolio, S. Aime, F. J. Vergeldt, H. Van As, Y. Yan, M. Drechsler, A. de Keizer, M. A. Cohen Stuart and J. van der Gucht, Chem. Commun., 2013, 49, 3736-3738.

38 Y. Yan, A. de Keizer, M. A. Cohen Stuart, M. Drechsler and N. A. M. Besseling, J. Phys. Chem. B, 2008, 112, 10908-10914.

39 J. Y. Wang, A. de Keizer, R. Fokkink, Y. Yan, M. A. Cohen Stuart and J. van der Gucht, J. Phys. Chem. B, 2010, 114, 8313-8319.

40 T. J. Prosa, B. J. Bauer and E. J. Amis, Macromolecules, 2001, 34, 4897-4906.

41 P. K. Maiti, T. Cagin, G. F. Wang and W. A. Goddard, Macromolecules, 2004, 37, 6236-6254.

42 Y. Yan, N. A. M. Besseling, A. de Keizer, M. Drechsler, R. Fokkink and M. A. Cohen Stuart, J. Phys. Chem. B, 2007, 111, 11662-11669.

43 R. Pecora, Nature (London), Phys. Sci., 1971, 231, 73-75.

44 H. C. Van der Hulst, Light Scattering by Small Particles, Wiley, New York, 1957.

45 The low generation dendrimers should also be present in other higher generations, i.e. the G5-G8, but their association-dissociation is probably obscured by the increase-decrease variation from the typical maximum-atPMC curve by the generation dendrimer under investigation.

46 A. W. Castleman and S. N. Khanna, J. Phys. Chem. C, 2009, 113, 2664-2675.

47 B. M. Rosen, D. A. Wilson, C. J. Wilson, M. Peterca, B. C. Won, C. H. Huang, L. R. Lipski, X. B. Zeng, G. Ungar, P. A. Heiney and V. Percec, J. Am. Chem. Soc., 2009, 131, 17500-17521.

48 R. J. Macfarlane, B. Lee, M. R. Jones, N. Harris, G. C. Schatz and C. A. Mirkin, Science, 2011, 334, 204-208.

49 A. Ruggi, C. Beekman, D. Wasserberg, V. Subramaniam, D. N. Reinhoudt, F. W. B. van Leeuwen and A. H. Velders, Chem.-Eur. J., 2011, 17, 464-467.

50 M. V. Gomez, J. Guerra, A. H. Velders and R. M. Crooks, J. Am. Chem. Soc., 2009, 131, 341-350.

51 J. During, A. Holzer, U. Kolb, R. Branscheid and F. Grohn, Angew. Chem., Int. Ed., 2013, 52, 8742-8745.

52 R. M. Crooks, M. Q. Zhao, L. Sun, V. Chechik and L. K. Yeung, Acc. Chem. Res., 2001, 34, 181-190. 